\title{
RETRACTED ARTICLE: Congenital Dermal Sinus with Triple Ostia: An Unusual Presentation
}

\author{
Kartik Chandra Mandal • Ram Mohan Shukla • Biswanath Mukhopadhyay
}

Received: 10 February 2012 / Accepted: 29 June 2012 / Published online: 18 July 2012

(C) Dr. K C Chaudhuri Foundation 2012

This article has been retracted by the journal (IJP) since plagiarism has been detected. The introduction and discussion is plagiarised to a great extent.

This article has been retracted by the journal (IJP) since plagiarism has been detected. The introduction and discussion is plagiarised to a great extent.

K. C. Mandal $\cdot$ R. M. Shukla $\cdot$ B. Mukhopadhyay

Department of Pediatric Surgery,

Nil Ratan Sircar Medical College and Hospital,

Kolkata, West Bengal, India

R. M. Shukla $(\bowtie)$

7E, Dinobandhu Mukherjee Lane, Sibpur,

Howrah 711102, West Bengal, India

e-mail: rammohanshukla@yahoo.co.in 